\title{
Paradoxical Deterioration During Anti-Tuberculous Therapy in Non-HIV- Infected Patients with Pleural Tuberculosis: A Pragmatic Approach
}

\author{
Luis Corral-Gudino, Alicia Rivas-Lamazares, Ana González-Fernández, Juan Carlos Hernando-García \\ Departamento de Medicina Interna, Hospital El Bierzo, Ponferrada, León, Spain
}

Received: 30/07/2016

Accepted: 09/08/2016

Published: 30/08/2016

How to cite this article: Corral-Gudino L, Rivas-Lamazares A, González-Fernández A, Hernando-García JC. Paradoxical deterioration during antituberculous therapy in non-HIV-infected patients with pleural tuberculosis: a pragmatic approach. EJCRIM 2016;3:doi:10.12890/2016_000475.

Conflicts of Interests: The Authors declare that there are no competing interests.

This article is licensed under a Commons Attribution Non-Commercial 4.0 License

\section{ABSTRACT}

We report a case of paradoxical deterioration. A male patient diagnosed with pleural tuberculosis, but who was not infected with human immunodeficiency virus (HIV), experienced clinical deterioration 3 weeks after the initiation of anti-tuberculous treatment. After other diagnoses were ruled out, a paradoxical response to treatment was established and the patient was started on systemic corticosteroids. Paradoxical response to treatment should be considered in patients with clinical deterioration after they start on anti-tuberculous treatment.

\section{LEARNING POINTS}

- The deterioration of patients with pleural tuberculosis during anti-tuberculous treatment should raise the suspicion of paradoxical response to treatment.

- Despite the clinical deterioration, patients should be kept on anti-tuberculosis treatment.

- There is no clear evidence concerning the benefits and harms of systemic corticosteroids or pleural drainage as additional treatment to control the patient's symptoms in this scenario.

\section{KEYWORDS}

Pleural tuberculosis, antitubercular agents, paradoxical response

\section{INTRODUCTION}

Clinical or radiological worsening of pre-existing tuberculous in a patient after they have start on specific treatment is defined as paradoxical response to treatment. Although paradoxical reactions are more common in human immunodeficiency virus (HIV)-infected patients, they can also be diagnosed in HIV-uninfected patients ${ }^{[1]}$. Paradoxical worsening of tuberculous pleural effusion is a rare condition with only a few cases published ${ }^{[2]}$.

Immunopathological damage as a consequence of an exaggerated and dysregulated host's inflammatory response or the excessive antigen load resulting from rapid mycobacterial lysis after initiation of treatment have been suggested as explanations for paradoxical worsening. It has not been established how clinicians should deal with such patients. In this paper we describe the case of a patient with a paradoxical response. In the discussion, we highlight the rationale for his differential diagnosis and the approach proposed for managing the patient's deterioration. 


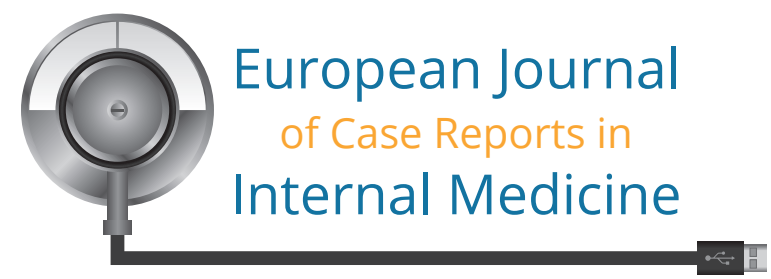

\section{CASE REPORT}

A 44-year-old man was referred to our clinic because of fever, arthralgia and myalgia. Clinical assessment, blood tests and chest x-ray were uninformative. The patient was prescribed symptomatic treatment and scheduled for another appointment 4 weeks later. On the second visit, patient reported shortness of breath and pleuritic chest pain on the left side where he had developed pleural effusion (Fig. 1). C-reactive protein (CRP) and erythrocyte sedimentation rate (ESR) were raised ( $23 \mathrm{mg} / \mathrm{l}$ and $49 \mathrm{~mm} / \mathrm{h}$ ). A thoracentesis showed a lymphocytic exudate with an adenosine deaminase (ADA) level of $50 \mathrm{IU} / \mathrm{I}$. A QuantiFERON-TB test was positive, while an HIV test was negative. The patient was diagnosed as likely having tuberculosis and started on isoniazid, rifampicin and pyrazinamide.

The patient still remained in poor health 4 weeks after starting treatment. He reported a slight clinical improvement during the first 3 weeks, but then began to feel even worse than before treatment initiation. On analysis his CRP and ESR were raised (106 mg/l and $67 \mathrm{~mm} / \mathrm{h}$ ), while his blood count, liver enzymes, urea, creatinine and electrolytes were normal. Pleural effusion remained similar on x-ray (Fig. 2).

Pleural aspiration was repeated showing non-complicated lymphocytic exudates. Due to his poor health, the patient was started on a short course of corticosteroids, with clinical improvement noted after the first week. The patient completed the 6-month treatment schedule for tuberculosis with complete clinical resolution of disease except for slight residual pleural thickening on chest x-ray (Fig. 3).

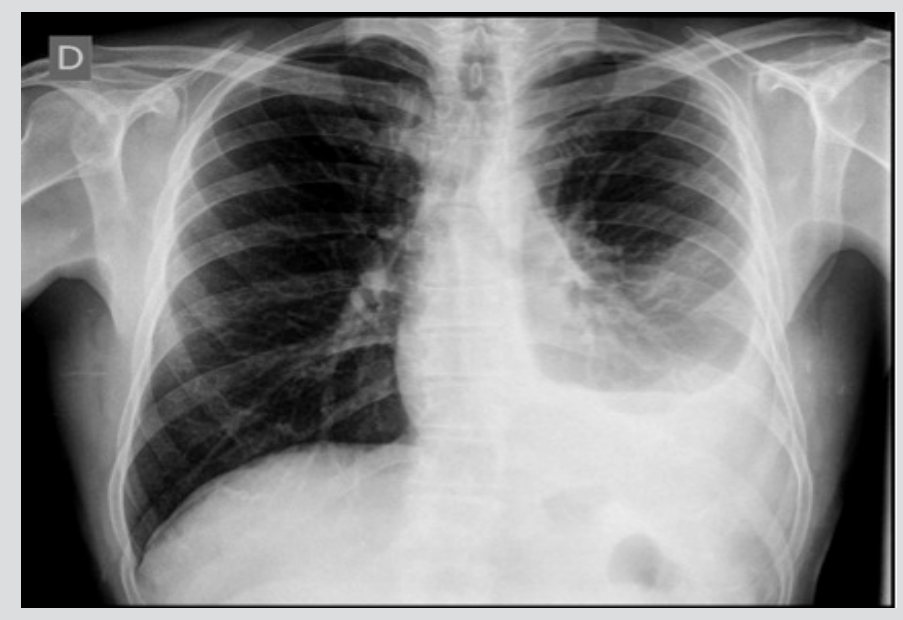

Figure 1.Chest $x$-ray before the patient started on anti-tuberculous treatment

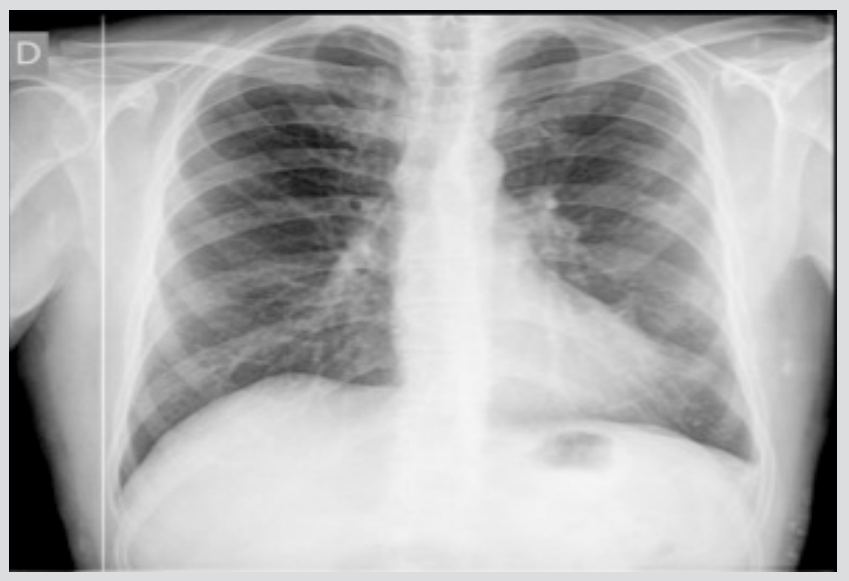

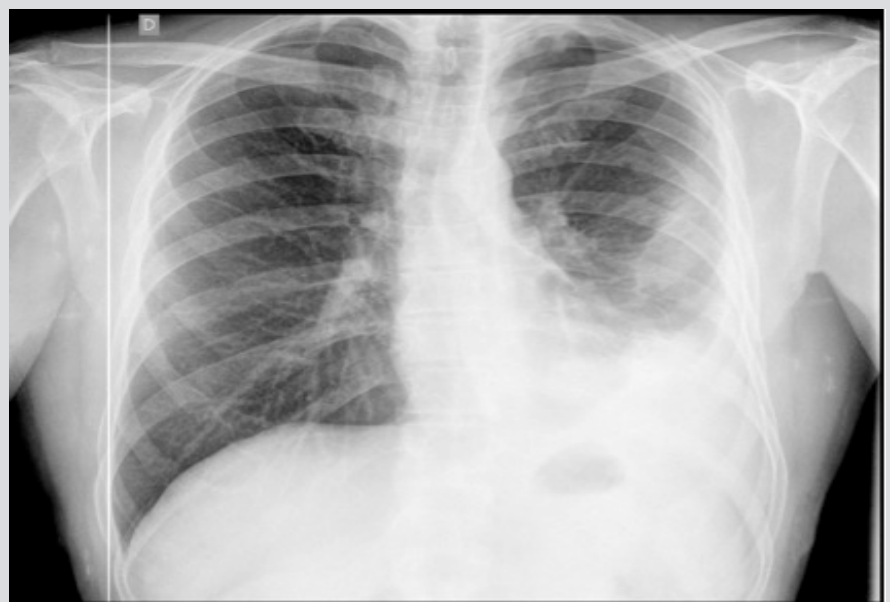

Figure 2. Chest $\mathrm{x}$-ray after 4 weeks of anti-tuberculous treatment

Figure 3. Chest $x$-ray 3 months after completion of anti-tuberculous treatment 


\section{DISCUSSION}

The deterioration of patients with pleural tuberculosis during treatment remains a challenge. The differential diagnosis should include: (i) treatment failure secondary to (a) inadequate anti-tuberculosis treatment, (b) poor adherence to the therapeutic schedule, (c) drug malabsorption, or (d) resistance to anti-tuberculosis drugs; (ii) side effects of anti-tuberculosis drugs; (iii) tuberculosis relapse; (iv) complications associated with pleural tuberculosis such as empyema; and (v) paradoxical deterioration. In addition, we need to definitively exclude other causes of lymphocytic pleural effusion such as malignancy or lymphoma, and other concurrent infections. A detailed clinical history focused on treatment adherence and likely treatment complications, and a new pleural aspiration are important for the differential diagnosis of suspected paradoxical response. In equivocal cases, a pleural biopsy may be required to exclude other diagnoses.

A systematic review summarized 143 episodes of paradoxical response in HIV uninfected patients ${ }^{[1]}$. The central nervous system was the most common site of initial involvement. The clinical signs and symptoms of paradoxical deterioration appeared in the initial site of infection in most patients. The most frequent manifestation in the respiratory system was the development or worsening of pleural effusion. In another case series ${ }^{[2]}$, paradoxical worsening of tuberculosis was detected in $16 \%$ of patients after treatment initiation. Dyspnoea was the main symptom of worsening, with massive effusion detected in $60 \%$ of these patients.

Currently, there are no guidelines regarding treatment for paradoxical response to pleural tuberculosis treatment. The patient should be maintained on standard specific therapy for 6 months. As an alternative approach, some authors prolong the course of tuberculosis treatment.

When a paradoxical response is suspected, the patient should be maintained on standard specific therapy for 6 months. In addition, to alleviate the patient's symptoms, three approaches are recommended: (i) the addition of symptomatic therapy, (ii) the addition of a short course of systemic corticosteroids, or (iii) complete drainage of pleural fluid. However, there is no evidence for any of these approaches in this setting, nor could evidence be extrapolated from patients with newly diagnosed tuberculosis. Corticosteroids can shorten the time to fluid resorption, but data are insufficient to determine the long-term outcomes ${ }^{[3]}$. Complete drainage of pleural fluid was associated with a significantly more rapid resolution of dyspnoea, but after the first week there were no differences ${ }^{[4]}$. One study showed residual pleural thickening was the same with or without drainage[4], while another showed less residual pleural thickening with thoracocentesis ${ }^{[5]}$. Therefore, corticosteroids and thoracocentesis should be restricted to selected patients who continue to have severe systemic symptoms such as fever or pain despite the use of anti-tuberculosis treatment and over-the-counter pain and fever medication, or in patients with severe pleural effusions causing dyspnoea.

In summary, the deterioration of patients with pleural tuberculosis during anti-tuberculosis treatment is a challenge. Lack of response to treatment, side effects of therapy, or the presence of an alternative diagnosis should be ruled out. The patient should be kept on standard anti-tuberculosis treatment. The decision to administer additional treatment to control the patient's symptoms should be based on the patient's preferences and physician's experience.

\section{REFERENCES}

1. Cheng VC, Ho PL, Lee RA, Chan KS, Chan KK, Woo PC, et al. Clinical spectrum of paradoxical deterioration during antituberculosis therapy in non-HIV-infected patients. Eur J Clin Microbiol Infect Dis 2002;21:803-809.

2. Al-Majed SA. Study of paradoxical response to chemotherapy in tuberculous pleural effusion. Respir Med 1996;90:211-214.

3. Engel ME, Matchaba PT, Volmink J. Corticosteroids for tuberculous pleurisy. Cochrane Database Syst Rev 2007;4:CD001876.

4. Lai YF, Chao TY, Wang YH, Lin AS. Pigtail drainage in the treatment of tuberculous pleural effusions: a randomised study. Thorax 2003;58:149-151.

5. Bhuniya S, Arunabha DC, Choudhury S, Saha I, Roy TS, Saha M. Role of therapeutic thoracentesis in tuberculous pleural effusion. Ann Thorac Med 2012;7:215-219. 